\title{
Research
}

\section{Reassured or fobbed off? Perspectives on infertility consultations in primary care:}

\author{
a qualitative study
}

\begin{abstract}
\section{Background}

Infertility affects $9 \%$ of couples in the UK. Most couples who visit their GP because they are worried about their fertility will ultimately conceive, but a few will not. Treatment usually happens in secondary care, but GPs can have an invaluable role in starting investigations, referring and giving support throughout treatment and beyond.
\end{abstract}

\section{Aim}

To inform clinical practice by exploring primary care experiences of infertility treatment among females and males, and discussing findings with a reference group of GPs to explore practice experience.

\section{Design and setting}

A qualitative patient interview and GP focus group study. Interviews were conducted in patients homes in England and Scotland; the focus group was held at a national conference.

\section{Method}

An in-depth interview study was conducted with 27 females and 11 males. A maximum variation sample was sought and interviews were transcribed for thematic analysis. Results were discussed with a focus group of GPs to elicit their views.

\section{Results}

Feeling that they were being taken seriously was very important to patients. Some felt that their concerns were not taken seriously, or that their GP did not appear to be well informed about infertility. The focus group of GPs highlighted the role of protocols in their management of patients who are infertile, as well as the difficulty GPs

faced in communicating both reassurance and engagement.

\section{Conclusion}

Simple things that GPs say and do, such as describing the 'action plan' at the first consultation, could make a real difference to demonstrating that they are taking the fertility problem seriously.

\section{Keywords}

infertility; primary care; qualitative; support.

\section{INTRODUCTION}

Estimates vary, but recent research suggests that $9 \%$ of couples are involuntarily childless, and at least onequarter of couples experience unexpected delays in achieving their desired family size., 1,2 Although individuals experience infertility differently, research shows that experiences can have lasting emotional effects. ${ }^{3,4}$ Unsuccessful treatment can negatively impact quality of life, leaving couples anxious and depressed with a sense of loss and bereavement. ${ }^{5-7}$ Even successful treatment can come at great emotional cost. ${ }^{8}$

Infertility treatment usually happens in secondary care. Research shows that three-quarters of couples presenting to their GP for the first time will be referred for specialist help. ${ }^{9}$ However, GPs have an important role in initiating tests for both partners, making timely referrals, and potentially offering ongoing support as couples go through treatment. ${ }^{10}$ Although clear guidelines exist on which tests and referrals to carry out, there is little guidance on the role primary care could have in information giving and emotional support.

The Royal College of General Practitioners has not issued any written guidance on managing fertility issues. GPs are likely to use guidelines from the National Institute for Health and Clinical Excellence (NICE), GP Notebook la respected website for GPs www.gpnotebook.co.uk), and NHS Clinical

L Hinton, BA (Hons), DPhil, senior qualitative researcher; S Ziebland, BA (Hons), MSc, reader in qualitative health research, Health Experiences Research Group, Department of Primary Care Health Sciences; JJ Kurinczuk, BSc (Hons), MSc, MD, FFPH, professor of perinatal epidemiology and director, National Perinatal Epidemiology Unit, University of Oxford, Oxford.

\section{Address for correspondence}

Lisa Hinton, University of Oxford, Health Experiences Research Group, Primary Health Care Sciences,
Knowledge Summaries la service that is commissioned and paid for by NHS Evidence, and provided by NICE www.cks.nhs.uk/home). NICE guidelines define infertility as 'failure to conceive after regular unprotected sexual intercourse for 2 years in the absence of known reproductive pathology', although they suggest couples should be offered investigations after 1 year. ${ }^{11}$

The NHS website, Clinical Knowledge Summaries, gives GPs guidance about tests they could initiate, and when to consider referral. Taylor suggested that prompt referrals and a systematic protocol of investigations leading to appropriate treatment can help alleviate some distress associated with fertility concerns. ${ }^{12}$

Outside these general guidelines, the literature on the management of infertility in general practice is small. ${ }^{13-15}$ Morrison et al explored patient perspectives on referrals and exposed shortcomings in the provision of written information and emotional support. ${ }^{16}$ Other studies exploring the GP's role in the emotional management of infertility found differing approaches. German studies highlighted the challenge that involuntary childlessness presents to GPs, in terms of specialist knowledge and helping couples deal with both the social isolation of treatment and the crisis of childlessness. ${ }^{17,18}$ Some GPs felt they should do all they possibly could for their patients: others felt it was the responsibility of infertility specialists and outside their area

23-38 Hythe Bridge Street, Oxford, OX1 2ET.

E-mail: lisa.hintonaphc.ox.ac.uk

Submitted: 14 October 2011; Editor's response: 13 December 2011; final acceptance: 15 February 2012

(c)British Journal of General Practice

This is the full-length article (published online 28 May 2012) of an abridged version published in print. Cite this article as: Br J Gen Pract 2012; DOI: 10.3399/bjgp12X649133. 


\section{How this fits in}

Although there are protocols guiding tests and referrals, there is little guidance for GPs on the emotional support and informational role that primary care migh play in the infertility consultation. Feeling that they were being taken seriously was very important to patients, but they did not always feel that this was the case. A GP focus group highlighted the role of protocols and the difficulties of communicating both reassurance and engagement. When faced with patients concerned about their fertility, GPs could demonstrate their interest by suggesting an action plan and flagging the patient's notes, while reassuring them that most couples will conceive without a need for referral.

of expertise. ${ }^{19,20}$ Patients also have different expectations - although some are disappointed if their GP does not make an effort to help them, others regard quick referral to specialists positively. ${ }^{21}$

Qualitative research can illuminate meanings and understandings. ${ }^{22}$ This article draws on in-depth interviews from a study of people's experiences of infertility, as well as their information and support needs. ${ }^{23,24}$ In addition to the patient interviews, a focus group of GPs explored doctors' perspectives on the accounts of primary care consultations. This analysis adds to the literature by considering perspectives from both sides of the general practice consultation.

\section{METHOD}

The qualitative design used in-depth audiorecorded interviews with 27 females and 11 males. Interviews were conducted from 2007 until 2009 in England and Scotland. The study aimed for a diverse, maximum variation sample of participants, who were going through infertility investigations, treatment, or had finished treatment. ${ }^{25}$ Variation was sought across age, diagnosis, NHS or self-funded treatment, outcome, and those living with or without children. With approval from Anglia and Oxford Multicentre Research Ethics Committee, participants were recruited through GP surgeries, specialist consultants, support groups, online newsletters, and word of mouth.

Participants were sent a patient information sheet, had the study explained to them, and signed a consent form before the interview. All interviews were audiorecorded, with informed consent. The checked transcript was sent back to responders for approval. The interviews had two parts:

- an unstructured narrative section, in which participants were asked to tell their own story, with as little interruption as possible, to capture their own accounts of infertility and highlight aspects that were important to them; and

- a series of prompts, used by the interviewer, to explore particular issues further in the semi-structured part of the interview.

The second section included questions about experiences of general practice care before, during, and after treatment. Interviews lasted between 45 minutes and 2 hours.

The interviews were transcribed verbatim and analysed thematically using the organisational support of NVivo 7 software. ${ }^{26}$ Transcripts were read and reread carefully by the interviewer and a coding framework was developed. A second researcher independently coded the first few interviews; results were compared and differences discussed, then the coding framework was revised and further developed. The codes for experiences of primary care were read and discussed by all three authors. A qualitative interpretative approach was taken, combining thematic analysis with constant comparison, looking for anticipated and emergent themes. ${ }^{27} \mathrm{~A}$ modified grounded theory approach, using the 'one sheet of paper' method, ensured that all of the coded extracts within the theme were included and compared in the analysis. ${ }^{28}$ This approach ensured that every instance and nuance was considered, including negative evidence ('deviant cases'). ${ }^{29}$

Qualitative data collection and analysis often proceed iteratively to achieve data saturation, with analytic categories saturated when data from new interviews does not add any more to the analysis. The themes discussed here emerged out of the analysis, and reflect experiences widely reported in the interviews. Experiences of primary care were raised in both the unstructured and prompted sections of the interviews. The female interviewer was conscious of interviewing males and females about a sensitive topic; she reflected on how her preconceptions and perspectives might influence the analysis. Critical distance was assisted by the narrative method and through the 
involvement of the second researcher and the co-author. Pseudonyms are used when presenting the data.

Findings from an initial analysis were presented at the South West Society of Academic Primary Care's conference on 5-6 March 2009 in Winchester. A group of 10 academic GPs - both male and female were invited to stay and discuss their reactions to the findings. The discussion was recorded, transcribed verbatim, and analysed as detailed above.

\section{RESULTS}

\section{Interviews}

Characteristics of patients who participated in the study are given in Table 1. Experiences of primary care were often raised in the narrative, as well as the semi-structured, section of the interviews. Although people described experiences of good and bad practical medical care in general practice, the emotional and psychological support and engagement (or not) of GPs stood out.

Expectations. For many of these, usually young, healthy individuals presenting with conception difficulties, it was the first time they had consulted about anything more serious than a cold or vaccination. They understood that the GP orders initial investigations and tests, and acts as gatekeeper to specialists. Many realised that they needed to try to conceive for at least 1 year before visiting their GP and had high expectations of the first consultation. For others, the consultation was the first step in acknowledging there was a problem; this was sometimes embarrassing or even frightening. Maggie, aged in her early 30s, described her shock at being referred to a specialist:

'I just thought there would be something simple that we had overlooked. There wasn't, and ... and they said we needed to see a specialist. Which was very, very scary. I remember at that point - I think this was the first of my bursting into tears in doctors' waiting rooms - I think the shock of it, and also it felt very serious all of a sudden. If we were seeing a specialist, a fertility specialist, then there was a problem with our fertility.' (Maggie)

However, expectations varied. Some people's expectations of their GP were more measured; some only expected a referral, and others understood there are limits to what their GP, or anyone else, could do. Sarah had endometriosis and had several failed attempts at in-vitro fertilisation (IVF); she was sanguine about what her GP could offer:

'You know, I am not really, it is not that I wanted more from them than they offered, but [3-second pause] there is a kind of a limit to ... yes, what anybody else can do for you really.' (Sarah)

The importance of being taken seriously. Participants described looking to their GP for advice, information, and support through all stages of treatment. They also valued feeling that their GP was interested and that their concerns were being taken seriously. This was evidenced by GPs appearing engaged, committing time, being open to further visits and questions, making swift referrals for tests, displaying knowledge about infertility, and offering information.

Jane, who went on to have successful IVF, described her GP's proactive and sympathetic approach:

'They listened, they were caring ... um ... you know and one, you know, as I said, if you had an idea about what you thought might be causing or it may be you should this, he didn't pooh-pooh it, he just said, "Fine. We will look at that." And ... um ... very quick about referrals and things like that, so yes. (Jane)

GPs who seemed familiar with infertility treatments were appreciated; likewise, some patients welcomed receiving sensible advice about options and being asked how they wanted to proceed with treatment. Marine, who conceived her daughter after four IVF cycles, described how she was warned by her GP about the long, potentially difficult, journey ahead and reminded that she could stop treatment at any point. She appreciated this engagement with her situation and the realistic assessment of the challenges ahead:

'You know, what she, yes that's it, she was really good, because we came after the year after the ectopic and said, "Okay we've tried ra ra ra". And she said, "That's fine. I'm going to refer you. This is what's going to happen. You need a sperm test. You go to see the infertility nurses." And then she said, "Remember this could be a long process and there's a lot of choices coming up that you need to make, and remember that at any point you can say no." So she was really good actually, yes, she was brilliant. $\mathrm{Hmm}$. Hmm.' (Marine)

Those with poor experiences of primary 
care said they felt 'fobbed off', dismissed, or that the GP was not interested and they had wasted their time. Martha, who experienced secondary infertility and ultimately had IVF to conceive her second child, described her GP's resistance:

'The kind of "Why are you here? You have already got one, you know, there shouldn't be a problem" kind of thing. And I think that I was just, you know, sort of shocked that they could overlook how completely destructive this was, you know, for all of us in the family and, to an extent, beyond it as well.' (Martha)

Being allowed time and being offered the opportunity to return to discuss the problem were both viewed by patients as an indication of being taken seriously by their GP. Those who felt they were not given the time they needed described feeling let down. Maggie explained why this was a problem during her first GP appointment:

And I guess that was something that I did find, find hard. Because at, we'd been trying for 2 years at this stage, I'd got a lot of questions to ask ... um ... and my doctor didn't have time to answer those questions ... We had this appointment with her that raised more questions than it answered. (Maggie)

Some felt that their GP did not know much about infertility, and should know more, given its prevalence:

For GPs and nurses, just get educated. When someone is there for a blood test for an IVF cycle and they have been trying for a family for 3 years. Telling that, "Ah it took me 3 months to fall pregnant with my second child, so I know how you feel" is really quite inappropriate. Um ... and GPs know nothing [about] infertility unless they have been, have some personal experience.' (Naomi)

Maggie found it difficult to understand what her GP was telling her and would have liked information pitched at the right level to be taken away and considered:

I think I would have liked my doctor to be able to, to maybe just give us some information to take home with us and digest. Both of us were sitting in that meeting room with the doctor. My husband would have his questions, I had my questions, she was trying to convey some information to us. There were words just flying about the place and, I think, you know. very few of them actually went in for any of us. In a way, I think it would have been good to have something ... written on paper that we could take away with us and digest, certainly about what our options were and the route we would be taking. Because we didn't have a huge amount of, well, fortunately neither of us have ever been particularly ill, so the whole process, the kind of, you know, GPs and nurses and consultants and hospital visits, was alien to us.' (Maggie)

Support from the GP. Several participants described their GPs as having an ongoing, supportive interest, even after referral to fertility specialists. Jane described her GP as 'very supportive'. Steve - who, together with his wife had several unsuccessful treatment cycles, and eventually adopted two boys - commented:

'We had a lovely old-fashioned Dr Finlay's Casebook kind of GP who was probably incredibly politically incorrect but he was actually also one of the most supportive medical staff that we came across. And he was genuine in his support and his irritation that there wasn't more help available to us from, in terms of funding and you know, why he couldn't maybe give us drugs on prescription ... so he was lovely.' (Steve)

Some participants felt that their GPs were aware that the consequences of infertility could endure after treatment has stopped. These doctors were seen as supportive and empathetic, helping people to pick up the pieces after failed treatment, which might include referral for counselling. In contrast, some females did not receive the support they wanted from their GP, especially after treatment failed. Carol, who had been having fertility treatment for 9 years, was disappointed that her GP did not investigate her recurring urinary tract infection. She felt no empathy for her worry that infections were contributing to her treatment failure:

'The last time I saw him I was having recurring urinary tract infections and I said that I was desperately worried, that I felt that this was impacting, firstly on the treatment, because some of them happened during my treatment, and he literally would not send me for more tests to see if there was any problems with my urinary tract, as to why I was susceptible to it. And I said, "Look, you know, I am spending thousands of pounds on this, if it was your money I am sure that you would want reassurance." And, literally, he just 


\section{Box 2. Questions posed during GP focus group}

Do you see infertility much in primary care practice?

Are there any resources that you are using now?

Are there any gaps that you are aware of in what you have to offer?

What do you think should be the role of primary care in infertility? didn't want to know and I ended up really screaming at him. I was so angry and I just walked out and I haven't been back since. And his last comment to me was, "Isn't it about time you thought about adopting now?" (Carol)

Joanna gave up on fertility treatment and adopted two girls. She felt that her GP demonstrated no empathy for her situation but, rather, seemed uncaring, making comments that she found inappropriate and upsetting:

'The other thing he said was, "Well you've had most of your life without children, why do you suddenly decide you want children?" And I just thought that was bizarre. So I would have liked a little bit more understanding.' (Joanna)

\section{GP focus group}

The focus group was presented with initial interview findings and members were then invited to discuss the findings and four further questions (Box 2).

The GPs, both males and females, reported a wide variation in the incidence of infertility in their practice: one (male, part time) GP thought he had probably only referred five people in 6 years, whereas another (female, part time) estimated a figure of 20-30 over a similar period. Another female GP estimated seeing one female patient a month who had fertility questions.

Protocols. Several GPs stressed how protocols drive their approach to patients who are potentially infertile, but said it was something that was often hard to explain. Although several GPs felt constrained by protocols however, others felt they could be used to reassure patients that steps were being taken within the necessary waiting period. One said she ignored the protocols:

'Sometimes what you can do with a protocol is say, "Well I can't refer you for 24 months, but meantime we can get the sperm test." Because sometimes that takes a bloke 6 months to get round to it ... And you get FSH [follicle stimulating hormone], LH [luteinising hormone], this, this this, and by the time you've got that 2 years has appeared and they feel that you are doing something because ... you're not fobbing them off but you are saying, "Listen, these are the rules but we can get these things and then bang on 2 years we can ...". (Male GP)

Offering reassurance and taking the patient seriously. The GPs discussed how to demonstrate that they are taking their patients seriously, while offering reassurance that the problem is likely to resolve without treatment. They will often see couples with early concerns about conception, who, with more time and knowledge about basic reproductive physiology, will conceive without needing a referral:

... you want to be reassuring, so if you can take the pressure off then some people become pregnant. It seems to me that when you do the referral you're taking the pressure off and that's what you want to precipitate the pregnancy. And it does seem to me that part of your role, just like making a cancer diagnosis - although you want to make that cancer diagnosis, you also want to reassure appropriately [that] the person hasn't got cancer. The same thing with the infertility thing, you want to reassure appropriately that the other person hasn't got some underlying fertility problems so that they are more likely to become pregnant. I just wonder how you do that effectively without falling into this trap of being told you're not taking them seriously? (Male GP)

There was agreement that offering support and reassurance was an appropriate role for GPs, and that perhaps the most effective way of communicating this was by drawing up an action plan and flagging the patients notes:

'I think one of the most important thing[s] when you first meet someone is to give them a plan ... Give them one of those nice little diary cards and let them keep a diary. I mean there are all sorts of things you can do.' (Female GP)

The GP's role. Although working within protocols, the GPs did see themselves as gatekeepers, initiating rigorous assessments, referring at the appropriate time, and offering support. They also agreed that appearing interested and sympathetic was important:

I'd certainly say, "Look I don't know the up- 
to-date things. This is something l've got to find out. Because lots of new techniques have come in and ... you know, some of the clinics are doing different things." And I think that's important that they know that you're actually interested. That's part of being interested.'

\section{DISCUSSION}

\section{Summary}

Some couples with infertility concerns expect a great deal from their GPs, others want a simple referral. In this study, people going through infertility treatment valued feeling supported, which was demonstrated through small gestures, such as displaying empathy, or a willingness to refer for counselling if treatment failed. People also wanted be taken seriously, which was shown by GPs appearing engaged, taking time to listen, offering an open door, and proposing clear action plans.

The GP focus group helped explain why patients sometimes feel 'fobbed off'. GPs are mindful that the majority of couples they see will have successful spontaneous pregnancies and were concerned when they realised that their attempts to reassure and to be non-alarmist could be interpreted as 'fobbing off'; given the perception from some patients that their GPs did not take them seriously, this is an interesting insight. The interview data suggest that taking an active role could include GPs being aware of their patient's specialist treatment, offering an open door, or making or offering referrals for counselling.

\section{Strengths and limitations}

A strength of this study is the fact that the interview findings were considered with a GP focus group, who could then make practical suggestions to improve the management of the infertility consultation. Holding a focus group after presenting the interview findings was a fruitful way of collecting data on primary care perspectives, but it cannot be claimed that this invited group of conference attendees was typical of primary care staff. The GPs were academic clinicians from one area of the country (the southwest of England). Further research with GPs could identify other ways to improve infertility management in primary care.

This qualitative interview study used careful analysis to explore patterns in people's accounts of their experiences. All studies based on self-reports (qualitative or quantitative) are constrained by their context, as well as recall bias. People interviewed for this study all went on from their initial GP consultation to have lengthy treatment; the perspectives of people who have concerns but conceive without needing treatment are not present.

\section{Comparison with existing literature}

As Himmel demonstrated, GPs often see the wide variety of experiences of involuntary childlessness that can be challenging to manage. ${ }^{17,18}$ However, these results confirm previous research - in addition to initial baseline tests and swift referrals, people are seeking emotional support from their GPs. ${ }^{30}$ At this nexus, there appears to be an important, possibly unfulfilled, role for GPs, particularly with regard to patients whose treatment is unsuccessful. Receiving support can help patients who are infertile deal with the social situation of childlessness.

Several studies have found that couples who are infertile report a lack of information from their GPs; ${ }^{31,32}$ part of the supportive role of GPs could include information giving. As many GPs will see patients who are likely to conceive without referral to specialist care, offering basic fertility information can be central to managing these patients' expectations. Although NICE guidelines note the potential value of the GP as a channel of understandable information, this clearly did not always happen.

In 1966, Jensen, a family doctor in Liverpool, argued that nowhere in the field of medicine is more tact, honesty, and sympathy needed than in the handling of a couple who may be infertile. ${ }^{33}$ Before the use of modern infertility treatment lor even clomiphene, a selective estrogen receptor modulator), doctors would have been able to offer patients only basic physiological information; nevertheless, the supportive and mentoring role that Jensen described remains relevant. He stressed the longterm role of the family doctor in supporting the emotional needs of couples who are infertile, recognising that childlessness can have lifelong effects.

This study's results support the conclusions of Wilkes et al, ${ }^{21}$ who found that couples had a good experience of infertility management when communication with each other and with the GP was strong, and when the GP had a 'special interest' in fertility issues; they also found that a patient-centered professional relationship fostered informed decision making. Patients in their study felt that GPs should perform the initial tests in a primary care setting, which can help reduce stress by being a familiar and less threatening environment than a secondary care setting. 


\section{Implications for practice}

Some GPs will rarely see couples who are going through infertility, whereas others will see a reasonable number. This study suggests that GPs could reassure couples by: showing an interest in their concerns; demonstrably writing out an action plan; flagging their notes, so their treatment can be followed up or questions raised at their next consultation; and giving, or suggesting how they can get, more information about infertility and possible treatments (for example, websites of the Human Fertilisation and Embryology Authority [www.hfea.gov.uk] and Infertility Network. GPs who have fertility consultations more regularly could also consider developing relationships with infertility clinics, perhaps, most appropriately, with the fertility nurses, so that they have up-to-date knowledge and can refer patients to individuals. 


\section{REFERENCES}

1. Boivin J, Bunting L, Collins JA, Nygren KG. International estimates of infertility prevalence and treatment seeking: potential demand for infertility medical care. Hum Reprod 2007; 22(6): 1506-1512.

2. Bhattacharya S, Porter M, Amalraj E, et al. The epidemiology of infertility in the north east of Scotland. Hum Reprod 2009; 24(12): 3096-3107.

3. Woollett A. Having children: accounts of childless women and women with reproductive problems. In: Phoenix A, Woollett A, Lloyd E (eds.). Motherhood: meanings, practice and ideologies. London: Sage, 1991: 47-65.

4. Wirtberg I, Moller A, Hogstrom L, et al. Life 20 years after unsuccessful infertility treatment. Hum Reprod 2006; 22(2): 598-604.

5. Hammarberg K, Astbury J, Baker HWG. Women's experience of IVF: a follow-up study. Hum Reprod 2001; 16(2): 374-383.

6. Lechner $L$, Bolman $C$, van Dalen A. Definite involuntary childlessness: associations between coping, social support and psychological distress. Hum Reprod 2001; 22: 288-294

7. Monga M, Alexandrescu B, Katz S, et al. Impact of infertility on quality of life, marital adjustment and sexual function. Urology 2004; 63(1): 126-130.

8. Redshaw M, Hockley C, Davidson LL. A qualitative study of the experience of treatment for infertility among women who successfully become pregnant. Hum Reprod 2007; 22(1): 295-304

9. Wilkes S, Chinn DJ, Murdoch A, Rubin G. Epidemiology and management of infertility: a population-based study in UK primary care. Fam Pract 2009; 26(4): 269-274

10. Balen AH, Rutherford AJ. Management of infertility. BMJ 2007; 335(7620): 608-611.

11. National Institute for Clinical Excellence. Fertility: assessment and treatment for people with fertility problems. London: NICE, 2004. http://www.nice.org.uk/CG011 laccessed 27 Apr 2012).

12. Taylor A. ABC of subfertility. Making a diagnosis. BMJ 2003; 327(7413): 494-497.

13. Souter V, Penney G, Gorman D. A survey of infertility practice in primary care in Scotland. Br J Gen Pract 1997; 47(424): 727-728.

14. Emslie C, Grimshaw J, Templeton A. Do clinical guidelines improve general practice management and referral of infertile couples? BMJ 1993; 306(6894): 1728-1731.

15. Morrison J, Carroll L, Twaddle S, et al. Pragmatic randomised controlled trial to evaluate guidelines for the management of infertility across the primary caresecondary care interface. BMJ 2001; 322(7297): 1-5.

16. Morrison C, Bhattacharya S, Hamilton M, et al. Initial management of infertility: an audit of pre-referrral investigations and exploration of couples' views at the interface of primary and secondary care. Hum Fertil (Camb) 2007; 10(1): 25-31.

17. Himmel W, Ittner $\mathrm{E}$, Kochen $\mathrm{M}$, et al. Management of involuntary childlessness. Br J Gen Pract 1997; 47(415): 111-118.

18. Himmel W, Ittner $\mathrm{E}$, Schroeter M, Kochen M. The many facets of involuntary childlessness in general practice. Scand J Prim Health Care 1999; 17(1): 25-29.

19. Wilkes S, Hall N, Crosland A, et al. General practitioner's perceptions and attitudes to infertility management in primary care: focus group study. J Eval Clin Pract 2007; 13(3): 358-363.

20. Ittner E, Himmel W, Kochen W. German family physicians' attitudes toward care of involuntarily childless patients. Fam Med 2000; 32(2): 119-125.

21. Wilkes $\mathrm{S}$, Hall N, Crosland A, et al. Patient experience of infertility management in primary care : an in-depth interview study. Fam Pract 2009; 26(4): 309-316.

22. Greenhalgh T, Hurwitz B. Narrative based medicine: dialogues and discourse in clinical practice. London: BMJ Books, 1998.

23. Hinton L, Kurinczuk J, Ziebland S. Infertility; isolation and the Internet: a qualitative interview study. Patient Educ Couns 2010; 81(3): 436-441.

24. Hinton L. Infertility in the internet age: how women and men find information and support during treatment for infertility and subfertility. DPhil Thesis. Oxford: University of Oxford, 2010

25. Coyne I. Sampling in qualitative research. Purposeful and theoretical sampling; merging or clear boundaries? J Adv Nurs 1997; 26(3): 623-630.

26. Richards TJ, Richards L. Using computers in qualitative research. In: Denzin NK, Lincoln YS (eds.). Handbook of qualitative research. Thousand Oaks, CA: Sage Publications, 1994

27. Green J, Thorogood N. Qualitative methods for health research. London: Sage, 2004

28. Ziebland S, McPherson A. Making sense of qualitative analysis: an introduction with illustrations from DIPEx (personal experiences of health and illness). Med Educ 2006; 40(5): 405-414.

29. Miles MB, Huberman AM. Qualitative data analysis. An expanded sourcebook 2nd edn. Thousand Oaks, CA: Sage Publications,1994.

30. Smith $L$. The role of primary care in infertility management. Hum Fertil (Camb) 2003; 6(Suppl1): 9-12.

31. Peddie V, van Teijlingen E \& Bhattacharya S. Ending in-vitro fertilisation. Women's perceptions of decision making. Hum Fertil 2004; 7(1): 31-37.

32. Porter $\mathrm{M}$, Bhattacharya $\mathrm{S}$. Helping themselves to get pregnant: a qualitative longitudinal study on the information-seeking behaviour of infertile couples. Hum Reprod 2008; 23(3): 567-572.

33. Jensen P. Childlessness and general practice. J R Coll Gen Pract 1966; 11 $150-165$. 